\section{Fractionation of Polystyrene by Gel Filtration}

IN a recent paper ${ }^{1}$, Porath and Flodin have described a simple and rapid method for the fractionation of water-soluble substances. In this method, which they call gel filtration, aqueous solutions of the substances are applied to the top of a column containing swollen insoluble grains of cross-linked dextrans and are eluted with water; fractions are collected from the bottom of the column in order of decreasing molecular weight. Porath and Flodin ascribe this phenomenon to a form of molecular sieving, the efficiency of which depends on the mesh size of the swollen cross-linked beads. For example, small molecules which penetrate the gel particles will be retarded, while large molecules, which are completely excluded from the gel phase, will migrate without retention in the interstitial fluid.

This communication reports some preliminary experiments in which this technique has been applied to a non-aqueous system, the fractionation of a sample of polystyrene in benzene solution.

Since polystyrene is soluble in non-aqueous solvents such as benzene it is necessary to use substances which swell in these solvents for packing the column. Insoluble cross-linked polystyrene $(P S X)$ beads were used, since it is comparatively easy to prepare such beads with different degrees of swelling (for example, by using various concentrations of the cross-linking agent, divinyl benzene) and thus gels with different mesh sizes. The beads were prepared by suspension polymerization ${ }^{2}$ and the small quantities of soluble polymer, which these beads contain, were removed by packing a column with swollen beads and eluting with benzene until no further polymer could be detected in the eluant.

A sample of polystyrene, having a wide distribution of molecular weights ${ }^{3}$, was fractionated by a typical chromatographic elution technique using $P S X$ beads of different degrees of swelling (measured by observing the swollen volume of a known weight of beads in the presence of excess benzene). The fractions were recovered by freeze-drying the benzene solutions at the water pump followed by drying to constant weight at $60^{\circ} \mathrm{C}$. and were characterized by measuring the specific viscosity $\left(\eta_{\mathrm{sp}}\right.$.) at one concentration (usually $0.4 \mathrm{gm} . / 100 \mathrm{ml}$.) in toluene at $30^{\circ} \mathrm{C}$.

In Fig. 1 the values of $\eta_{\mathrm{sp}}$./c. are plotted against the cumulative weight together with two reference standards : (a) the horizontal line, which is $\eta_{\mathrm{sp}} / \mathrm{c}$. for the whole polymer and represents no fractionation ; (b) a plot of the results obtained from a conventional fractionation using the solvent volatilization method ${ }^{3}$.

It will be seen that whereas PSX beads which swell only $2 \cdot 2$ times give practically no fractionation, with beads having higher degrees of swelling fractionation appears in the lower molecular weight region. In the case of those that swell 51 times, the resolution in this region approaches that of the solvent volatilization method. However, the degree of fractionation in the high molecular weight region is much less pronounced, indicating that even with these beads the degree of cross-linking is not loose enough to allow the larger molecules to penetrate the network.

These preliminary experiments have established, therefore, that high polymeric materials, such as polystyrene, can be fractionated by this technique, and it is thought that, by preparing $P S X$ beads

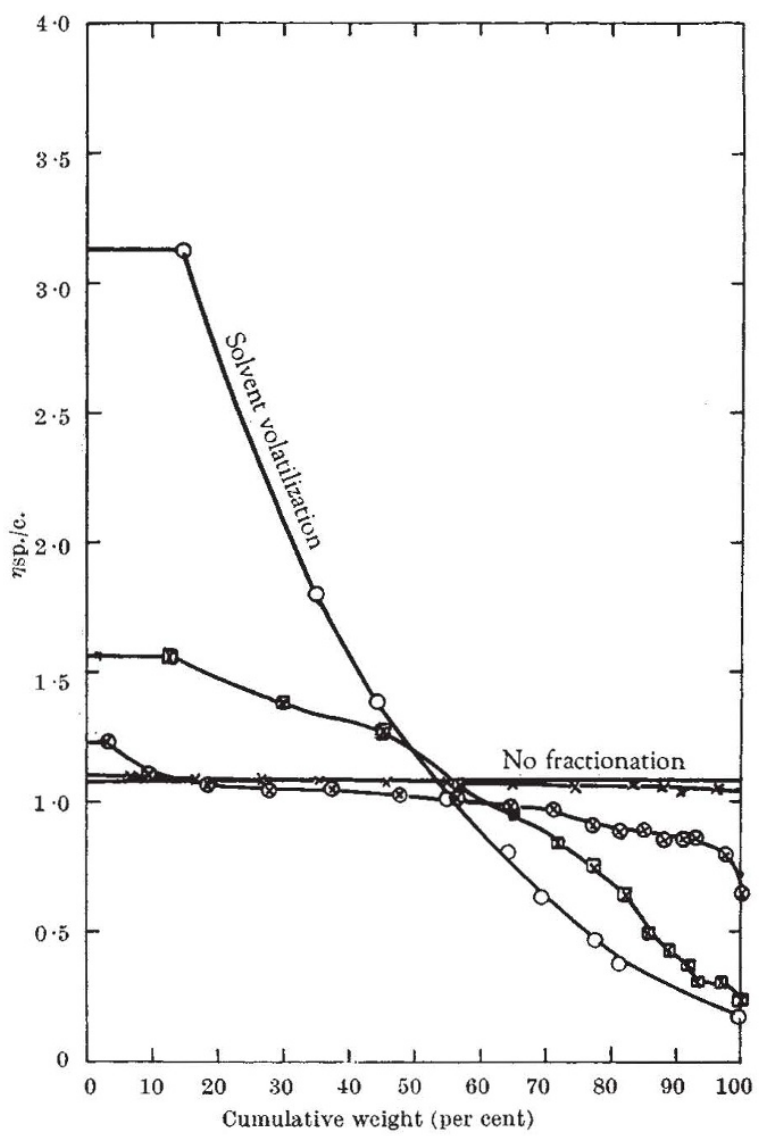

Fig. 1. Fractionation with cross-linked polystyrene beads swelling $13 ; \square, P S X / S 2$, degree of swelling 51

having a higher degree of swelling and/or by modifying the column technique, the efficieney can be improved to approach that of the conventional fractionation methods. The possibility arises, therefore, of developing a simple method of polymer fractionation in which the fractions can be collected automatically and which is economical in the use of solvents, since most of the benzene can be recovered during the freeze-drying.

A further possible development could be in the separation of very low molecular weight materials (cf. Porath and Flodin, who cite the separation of glucose from dextran fractions of $\bar{M}_{n} \quad 1,000$ and 20,000 ), since although practically no fractionation took place with the $P S X$ beads which swell $2 \cdot 2$ times, a little did, in fact, occur, suggesting that the smallest polystyrene molecules enter the PSX beads.

I wish to thank Miss S. A. Monksfield and Miss H. M. Sankey for assistance with the experimental work and Mr. D. Reichenberg for supplying the PSX beads 509 and 524, and also for his advice on the column procedure. This work is published by permission of the Director of the National Chemical Laboratory.

National Chemical Laboratory,

M. F. VAUGHAN

Department of Scientific and Industrial Research, Teddington, Middlesex.

1 Porath, J., and Flodin, P., Nature, 183, 1657 (1959).

2 Pepper, K. W., J. App. Chem., 1, 124 (1951).

3 Green, J. H. S., and Vaughan, M. F., Chem. and Indust., 829 (1958). 